\title{
Energy Efficient and High Capacity Tradeoff in Distributed Antenna System for a Green Cellular Network
}

\author{
Mehran Behjati, Mohammed H. Alsharif, Rosdiadee Nordin, and Mahamod Ismail \\ Department of Electrical, Electronic and System Engineering, Universiti Kebangsaan Malaysia, 43600 Bangi, Selangor, Malaysia \\ Correspondence should be addressed to Mehran Behjati; mbehjati@eng.ukm.my
}

Received 10 November 2014; Revised 3 January 2015; Accepted 4 January 2015

Academic Editor: Rui Zhang

Copyright ( 2015 Mehran Behjati et al. This is an open access article distributed under the Creative Commons Attribution License, which permits unrestricted use, distribution, and reproduction in any medium, provided the original work is properly cited.

\begin{abstract}
Two main concerns for designing a wireless system are more network capacity and less energy consumption. Recently, distributed antenna system (DAS) has received considerable attention due to its potential to provide higher spectral efficiency (SE) and uniform coverage for cellular networks. In this regard, this paper compares the performance of DAS with centralized antenna system (CAS) in LTE-A system in terms of energy efficiency (EE), where practical restrictions such as out-of-cell interference, path loss, and small scale fading are taken into account. Furthermore, the EE and system power consumption are investigated under three different cell-load scenarios (high, moderate, and low load) where different numbers of antennas are activated and remaining of antennas are under sleep mode. Finally, based on the tradeoff between power-saving and EE, two optimal DAS antenna deployments are proposed for low and moderate cell-load scenarios. The results reveal that DAS considerably outperforms CAS in terms of EE and by optimal deploying antennas of DAS significant power-saving and EE are achievable. The proposed methodology achieved savings of up to $27.63 \%$ in terms of energy savings in a macrocell with guarantee of a high capacity of data.
\end{abstract}

\section{Introduction}

The sudden increase in subscribers and demand for highspeed data has prompted cellular operators to increase the number of base stations (BSs) to fulfil the needs of mobile subscribers. This increase has subsequently increased the overall energy consumption, operational costs, and carbon footprint of cellular networks. Accordingly, improving the energy efficiency (EE) of wireless networks has become a compelling challenge for researchers, vendors, and mobile operators, not only to reduce the operational costs but also to reduce the global carbon emissions. The focus is on creating green cellular networks at BSs because in real mobile networks most of power consumed by BSs, which is approximately $57 \%$ of the total energy consumption [1]. While [2] reported that the total energy consumed by the internet communication technology (ICT) takes more than three percent of the worldwide electric energy consumption, this portion is going to increase in the future. A number of interesting works have been carried out to address the issue, by implementation of "greener" cellular networks that are less expensive to operate. Overall, these improvements can be achieved through two approaches. The first approach is to reduce the power consumption of BSs using powerefficient hardware. The second approach is to adopt the intelligent management of network elements based on traffic load variations [3], which is the focus of this work.

Spectral efficiency (SE) and EE are two key figures of merit to consider the performance of wireless communication networks. It is investigated in [4] that the SE of cellular networks must be increased from $0.5-2.5 \mathrm{~b} / \mathrm{Hz}$ (in 2010) to $5-$ $20 \mathrm{~b} / \mathrm{Hz}$ (in 2015). Therefore some technologies are required to enhance the SE and capacity for cellular networks. In this regard, LTE and LTE-A systems are designed to operate in midhigh SE region [5]. In conventional cellular system, all antennas are colocated at the central BS, which is known as centralized antenna systems (CASs). In such systems, by increasing the distance between user equipment (UE) and BS, transmitted downlink signal exponentially attenuated and most portion of signal power wasted which results in poor network coverage.

On the other hand, by increasing the transmit power sever interference is experienced by users especially at the cell-edges. Consequently, system performance degrades in 
such system, while distributed antenna system (DAS) is one of the upcoming technologies, which improves coverage and capacity for both hot and dead spots without the need for extensive base station deployments. Therefore, recently applying DAS technology in LTE and LTE-A has drawn much attention.

In DAS some of the antennas are located at the central BS and other antennas are distributed throughout the cell which is known as radio remote units (RRUs). The RRUs have minimal intelligence of their own and controlled by the central BS and contain a low-complex processor, up/downconverters, and low noise amplifiers [6] and are connected to the central BS via high-bandwidth low-latency dedicated connections such as fiber optics [7]. In contrast to CAS, DAS reduces the access distance (between UE and BS) therefore reducing the interference by minimizing the transmit power (while channel quality is kept the same). DAS provides uniform coverage [8], enhances capacity [9] and area SE [10], mitigates path loss and shadowing effects, and reduces outage on the downlink [11]. Results in [9] show that DAS reduces other-cell interference in a multicell scenario and significantly improves throughput especially for users near cell-edges, while outof-cell interference is the main restriction factor to achieve system capacity. The results in [12] show a lower power consumption of DAS on the uplink versus CAS. The current perspective of DAS and its application to the downlink of cellular systems can be found in [8].

Moreover, multiple-input multiple-output (MIMO) is a well-known and promising technology which can improve the SE and throughput for wireless communication systems [13]. In this regard, the application of MIMO to DAS is a topic of ongoing research where recent works in $[10,14-$ 16] investigated the performance of (multiuser) MIMO in DAS and stated that MIMO is an effective and promising strategy for DAS, which provides higher data rate for system, while authors of [17] compared the EE of MIMO and single input single output (SISO) in CAS over different transmission distances. The comparison shows that, in CAS, MIMO is not always more EE than SISO especially at a short distance. Therefore, the fundamental issues of this paper are as follows: how to exploit MIMO technique to optimize energy consumption in the network and how to exploit DAS in LTE-A system in such way that operates in EE powersaving tradeoff regime. In this regard, some techniques are well-known to enhance power-saving in the cellular network such as switching off cells, reducing number of BS's antenna, and reducing cell bandwidth. In the cellular network, power amplifier (PA) consumes most of power in BSs. Therefore, to reduce the power consumption of network (while the number of BSs is very large), one effective solution is reducing the number of antennas (switching off the PAs) based on the traffic load conditions, which is the focus of our work, since each PA roughly consumes the largest portion of energy in a BS (approximately $65 \%$ of the total energy consumption).

In this regard, less attention has been paid to investigate the EE and power-saving for DAS. Authors of [18] investigated the EE and SE of CASs in multicell and multiantenna scenarios. Performance of DAS and CAS was compared in [4] that exploits that DAS can provide significant enhancement in transmit power efficiency. By considering EE and SE of DAS, the optimal location and number of RRUs were investigated in [19]. An optimal power allocation method was proposed in [20] to maximize EE for DAS. EE and SE of DAS were investigated in $[5,6,21,22]$ which reveal remarkable performance of DAS versus CAS; nevertheless impact of outof-cell interference was ignored.

To investigate the performance of DAS, it is important to consider the out-of-cell interference where RRUs are distributed throughout the cell and impacts the signal to interference plus noise ratio (SINR) level. Moreover, there is a lack in investigation the performance of DAS under some realistic channel parameters such as path loss, fading, and shadowing. In this regard, this paper concentrates to investigate the performance of DAS under practical constraints such as out-of-cell interference, path loss, and smalland large-scale fading, where a LTE-A standard compliant simulator is utilized. Moreover all results are provided under considerations of practical system parameters such as user scheduling, received antenna combiner, and precoding transmission. To obtain the optimal network architecture for DAS (in terms of EE), performance of DAS with different antenna configurations is investigated and compared with CAS. Moreover, EE of DAS is investigated under different antenna architectures, cell-loads, and channel bandwidths. Furthermore, the system power consumption is investigated in the three different cell-load scenarios: high, moderate, and low load, where different system architectures with different antenna deployments are examined. Finally, by making tradeoff between EE and power-saving in two sleep modes, low and moderate load scenarios, optimal antenna deployments are proposed for DAS.

The rest of the paper is organized as follows. In Section 2, the system model is described. Section 3 discusses the methodology. The results and discussion are presented in Section 4 and Section 5 concludes the paper.

\section{System Model}

2.1. Downlink Model. Figure 1 depicts the architecture of considered DAS with $I$ cells which each cell $i=\{1, \ldots, I\}$ contains one central BS with $M_{c, i}$ transmit antennas and $R_{i}$ RRUs which each of them equipped with $M_{r, i}$ antennas, where $r \in\left\{1, \ldots, R_{i}\right\}$. The total number of served users in cell $i$ is $K_{i}$ and each user $k \in\left\{1, \ldots, K_{i}\right\}$ is equipped with $N_{k, i}$ received antennas. The input-output relationship on $k$ th user in cell $i$ is given by

$$
\begin{aligned}
\mathbf{y}_{k, i}= & \mathbf{H}_{k, i} \mathbf{x}_{k, i}+\mathbf{H}_{k, i} \sum_{u=1, u \neq k}^{K_{i}} \mathbf{x}_{u, i} \\
& +\sum_{j=1, j \neq i}^{I} \mathbf{H}_{k, i}^{(j)} \mathbf{X}_{j}+\mathbf{n}_{k, i},
\end{aligned}
$$

where $\mathbf{y}_{k, i}$ is the perturbed received signal vector to user $k$ in cell $i$ and $\mathbf{H}_{k, i}^{(j)}$ is the channel matrix between user $k$ in cell $i$ and all transmit antennas of cell $j, M_{j}$ as

$$
M_{j}=M_{c, j}+\sum_{r=1}^{R_{i}} M_{r, j}
$$




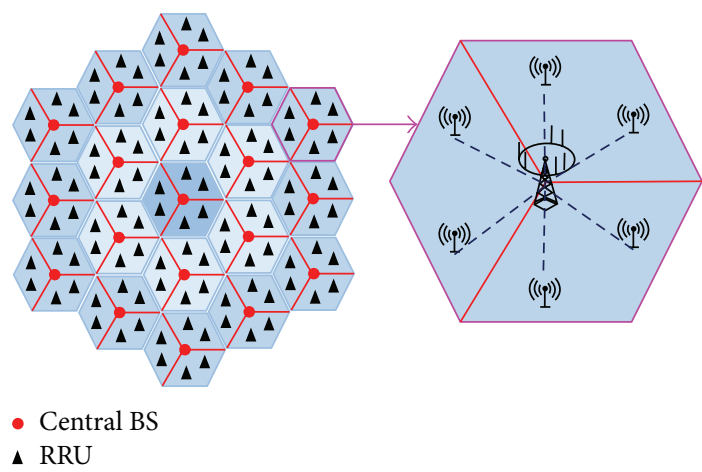

FIGURE 1: Example of considered DAS, consisting of 19 cells with 22/1 configuration. Each cell contains one sectorized central BS which each sector equipped with 2 central-antennas and 2 RRUs that each of which equipped with one antenna and connected to the central $B S$ via fiber optic.

$\mathbf{n}_{k, i} \sim N\left(0, \sigma_{n}^{2} \cdot I\right)$ is additive white Gaussian noise (AWGN). $\mathbf{x}_{k, i}$ is the scheduled and precoded symbol vector, and $K \subseteq$ $\left\{1, \ldots, K_{i}\right\}$ is the set of selected users that are served in parallel over a given time-frequency resource in cell $i$. Here proportional fair scheduler is employed, whereby users are selected based on a greedy algorithm, where BS uses the available SINR value and searches through the unscheduled users to select users which can maximize sum-rate (for original definition see Algorithm 2 in [23]). Prior to transmit user symbol vector, $\mathbf{s}_{k, i}$ over the downlink channel, it should be precoded with precoder vector, $\mathbf{f}_{k, i}$ to map the transmit symbol vector onto the $M_{i}$ transmit antennas and allocates the available transmit power $\left(P_{\mathrm{tx}}\right)$ among users, $p_{k, i}$ (for details refer to section 2.2 in [16]). Therefore,

$$
\begin{gathered}
\mathbf{x}_{k, i}=\mathbf{f}_{k, i} \mathbf{s}_{k, i}, \\
\mathbf{F}_{i}=\widehat{\mathbf{H}}_{k, i}^{H}\left(\widehat{\mathbf{H}}_{k, i} \widehat{\mathbf{H}}_{k, i}^{H}\right)^{-1} \operatorname{diag}\left(p_{k, i}\right)^{1 / 2},
\end{gathered}
$$

where $\mathbf{F}_{i}=\sum_{k \in K} \mathbf{f}_{k, i}$ is the precoding matrix.

To extract the multiplexing gain, MU-MIMO technique is utilized, where multiusers are served in parallel over a given time-frequency resource. Although transmission strategy such as multiple streams per users is possible, it has been shown in [24] that schemes such as zero-forcing (ZF) [25] which transmits single stream per user exploit more multiuser diversity gain.

By distributing RRUs throughout the cell, different path losses are experienced. Therefore, the channel matrix should be decomposed as follows [16]:

$$
\mathbf{H}_{k, i}^{(j)}=\overline{\mathbf{H}}_{k, i}^{(j)} \mathbf{C}_{k, i}^{(j)^{1 / 2}},
$$

where $\overline{\mathbf{H}}_{k}$ is small-scale fading channel matrix, where for Rayleigh fading channel its elements are independent identically distributed $\mathcal{N}(0,1) . \mathrm{C}_{k, i}^{(j)}$ is the channel gain matrix, which characterizes large-scale shadow fading and path loss with respect to the different RRUs, where

$$
\mathbf{C}_{k, i}^{(j)}=\operatorname{diag}\left(\left[\gamma_{k, i}^{(1, j)}, \gamma_{k, i}^{(2, j)}, \ldots, \gamma_{k, i}^{\left(M_{j}, j\right)}\right]\right),
$$

where $\gamma_{k, i}^{(n, j)}$ is the large-scale channel gain between transmit antenna $n$ in cell $j$ and received antenna of user $k$ in cell $i$

$$
\gamma_{k, i}^{(n, j)}=10^{-L_{k, i}^{(n, j)} / 10}
$$

where $L_{k, i}^{(n, j)}$ is the macroscale path loss between antenna $n$ in cell $j$ and user $k$ in cell $i$

$$
L_{k, i}^{(n, j)}=\max \left(70,128.1+37.6 \log _{10}\left(\frac{d_{k, i}^{(n, j)}}{1000 \mathrm{~m}}\right)\right),
$$

where $d_{k, i}^{(n, j)}$ is the distance between user $k$ in cell $i$ and transmit antenna $n$ in cell $j$.

2.2. Power Model. Power consumption can be decomposed into two parts: fixed and dynamic. The fixed part is the baseline BS power consumption (e.g., signal processing, site cooling, power supply, and battery backup), which depends on both the hardware and software configurations of the BS and it is independent of the traffic load, whereas the dynamic part accounts for the power consumed in RF transmission and depends on the traffic load. The total power consumption is calculated as [26]

$$
P_{\text {tot }}^{\text {macro-BS }}=N_{\mathrm{TRX}}\left[\frac{P_{\mathrm{tx}} / \eta_{\mathrm{PA}}\left(1-\sigma_{\mathrm{feed}}\right)+P_{\mathrm{RF}}+P_{\mathrm{BB}}}{\left(1-\sigma_{\mathrm{DC}}\right)\left(1-\sigma_{\mathrm{MS}}\right)\left(1-\sigma_{\mathrm{cool}}\right)}\right] \text {, }
$$

where $\sigma_{\text {feed }}, \sigma_{\mathrm{DC}}, \sigma_{\mathrm{MS}}$, and $\sigma_{\text {cool }}$ denote losses incurred by the feeder, DC-DC power supply, main supply, and cooling, respectively. $P_{\mathrm{tx}}, P_{\mathrm{RF}}$, and $P_{\mathrm{BB}}$ are the output power per transmit antenna, radio frequency, and baseband power, respectively. $\eta_{\mathrm{PA}}$ denotes the PA power efficiency, and $N_{\mathrm{TRX}}$ is the number of transceiver, which can be computed as follows:

$$
N_{\text {TRX }}=N_{\text {Carriers }} \times N_{\text {Sectors }} \times N_{\text {Antennas }},
$$

where $N_{\text {Carriers }}, N_{\text {Sectors }}$, and $N_{\text {Antennas }}$ denote the number of carriers, sectors, and antennas, respectively.

The total daily consumed power in our studied system can be computed as

$$
P_{\text {Cons }}^{\text {day }}=\left[\mathrm{hr}_{\text {high }}+\mathrm{hr}{ }_{\text {mod }}+\mathrm{hr}_{\text {low }}\right] \times(a),
$$

where $\mathrm{hr}_{x}$ refers to the number of hours per day in which an antenna is activated in three different cell-load scenarios (high, moderate, and low load) where it will be discussed in more detail in Section 3. $a=N_{\text {ant }}^{\text {active }} \times P$, where $N_{\text {ant }}^{\text {active }}$ refers to the number of activated antennas and $P$ is the total consumed power as (8).

2.3. Throughput Model. To achieve the throughput, the achievable mutual information introduced in [27] is used

$$
L=\max _{F \in \mathscr{F}} \sum_{k=1}^{N_{\text {tot }}} Z B_{\text {sub }} \log _{2} \operatorname{det}\left(\mathbf{I}_{N}+\frac{1}{\sigma_{n}^{2}} H_{k} \mathbf{F F}^{H} \mathbf{F}_{k}^{H}\right),
$$

where $N_{\text {tot }}$ is the total number of usable subcarriers, $\mathscr{F}$ is the set of precoding matrices, $I_{N}$ is an identity matrix of size of 
$N$, and $\sigma_{n}^{2}$ is the energy of the noise and interference at the receiver. $B_{\text {sub }}$ is the bandwidth of a subcarrier, given as

$$
B_{\text {sub }}=\frac{N_{s}}{T_{\text {sub }}-T_{\mathrm{cp}}},
$$

where $N_{s}=14$ is the number of OFDM symbols in one subframe, $T_{\text {sub }}=1 \mathrm{msec}$ is the duration of one subframe, and $T_{\mathrm{cp}}$ is the time required for the transmission of all cyclic prefixes within one subframe. $Z$ is a factor of the system losses due to the transmission of the reference symbols and the cyclic prefix

$$
Z=\frac{T_{\mathrm{sub}}-T_{\mathrm{cp}}}{T_{\mathrm{sub}}} \cdot \frac{N_{\mathrm{sc}} \cdot N_{s} / 2-N_{\mathrm{ref}}}{N_{\mathrm{sc}} \cdot N_{s} / 2},
$$

where $N_{\mathrm{sc}}=12$ is the number of subcarriers in one resource block and $N_{\text {ref }}$ is the number of reference symbols per resource block.

2.4. Energy Efficiency (EE). The number of bits transmitted per Joule of energy, called energy effciency (EE), is used as a performance measure, which is defined as [28]

$$
\mathrm{EE}=\frac{L}{P_{\mathrm{tx}}}
$$

\section{Methodology}

In this study, results are provided based on computer simulation and mathematical analysis. To simulate the results, the Vienna LTE-A link-level simulator [27] is used which is compliant to the LTE-A specifications. According to the remarkable impact of out-of-cell interference on the DAS, multicell scenario is considered. System level simulation of multicell MU-MIMO scenario requires detailed knowledge of physical layer for downlink transmission and feedback procedure, which results in massive computational complexity. Therefore, Vienna simulator uses the hybrid link/system level simulations to consider the physical details for one cell (central cell) and rest of cells are considered as out-of-cell interference (interference model can be found in [10]). Since Monte Carlo simulations are performed, the cell throughput is evaluated in terms of $95 \%$ of empirical cumulative density function of (11) and EE is computed according to (14).

The simulation parameters under considerations are listed in Table 1. Figure 1 illustrated the considered cellular network, where central cell is surrounded with two interfering BSs tiers, and all cells are configured as DASs. Each cell is covered by one central BS and $R_{i}$ uniformly distributed RRUs which located equiangularly on a ring with radius of $(2 / 3) \mathrm{x}$ cell radius. According to (2) the total number of transmit antennas is shared among the central BS and RRUs. The central BS is equipped by sectorized antennas $\left(3 \times 120^{\circ}\right)$ and RRUs are equipped by omnidirectional antennas. Users are randomly distributed in the central cell and the number of transmit antennas and RRUs are determined based on the cell-load densities.

For downlink transmission, only one antenna with best channel quality is selected which results in lower interference
TABLE 1: Simulation parameters [27].

\begin{tabular}{lc}
\hline Parameters & Value \\
\hline Carrier frequency & $2.6 \mathrm{GHz}$ \\
Subcarrier bandwidth & $15 \mathrm{kHz}$ \\
Noise power spectral density & $-174 \mathrm{dBm} / \mathrm{Hz}$ \\
Maximum power & $46 \mathrm{dBm}$ \\
Shadowing standard deviation & $4 \mathrm{~dB}$ \\
Channel model & Temporally correlated \\
& block fading frequency flat \\
CSI feedback & Rayleigh fading \\
Received antenna combiner & Perfect \\
User scheduler & MMSE \\
Transmission strategy & Greedy scheduler \\
Number of BSs & Algorithm 2 in [23] \\
Cell radius & ZF beamforming \\
Number of UE's antennas & 19 \\
UE speed & $500 \mathrm{~m}$ \\
Number of UEs in high load & 1 \\
scenario & $3 \mathrm{Km} / \mathrm{h}$ \\
Number of UEs in moderate load & 36 \\
scenario & 18 \\
Number of UEs in low load scenario & 6 \\
Number of simulation positions & 20 \\
\hline
\end{tabular}

and higher downlink performance [9]. Furthermore, each UE is connected just to a RRU that provides the highest EE [5].

The configurations of DAS are denoted as $M_{c, i}-R_{i} / M_{r, i}$, for example, 2-6/1 means 2 antennas are allocated at the central BS and there are 6 RRUs that each of which is equipped with 1 antenna. The total transmission power, $P_{\mathrm{tx}}$, is shared among the central BS and RRUs as

$$
\sum_{i=0}^{j} p_{i}=P_{\mathrm{tx}}
$$

where index $i=0$ is for central BS and $i=\{1, \ldots, j\}$ is for RRUs. Therefore, based on the number of RRUs, the power is shared among transmitter antennas, for example, in the 2-6/1 scenario $p_{0}=0.4 \times P_{\mathrm{tx}}$ and $p_{i}=0.1 \times P_{\mathrm{tx}}$ where $i=\{1, \ldots, 6\}$.

Generally, the infrastructures of cellular networks are designed to support daytime traffic. Traffic loads during the day differ from those at night. Hence, one cannot ignore the amount of energy that is wasted due to the lack of efficient use of resources, particularly for low loads. Herein, according to the results of [29], three different cell-load scenarios are assumed: (i) normal case at a high traffic load $(0.8<\lambda)$, where the period of time for this case ranging from 9:00 to 21:00; (ii) moderate traffic load $(0.3 \leq \lambda \leq 0.8)$, where the period of time ranging from 7:00 to 9:00 and from 21:00 to 0:00; (iii) low traffic load $(0<\lambda<0.3)$ and the period of time from 0:00 to 7:00. Figure 2 summarizes the three aforementioned cases.

In order to reduce the daily system power consumption, we propose two sleep modes: one for low load case and 


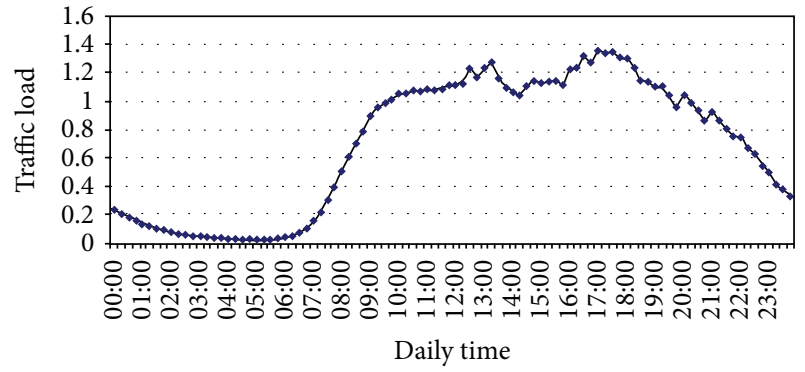

Figure 2: Average traffic distribution over a day for urban environment [29].

another for moderate load scenario. Therefore, it is assumed that the numbers of activated antennas are changed three times a day. To find the optimal number of activated antennas (DAS configuration), for two sleep modes, the objective is to jointly maximize the EE and power-saving for DAS. To do so, the antenna switch-off decision is determined based on two considerations. The first consideration meets the need of subscribers where it provides a high data rate according to the traffic load. The second consideration is that the largest possible number of antennas should be switched off to obtain the greatest decrease in energy consumption. The following two steps describe the considerations in detail.

Step 1 (optimal number of antennas for EE maximization (minimum EE degradation under sleep modes)). In order to maximize the EE in (14), on the one hand, the system throughput, $L$, has to be maximized. On the other hand, the transmit power, $P_{\mathrm{tx}}$, in the denominator has to be minimized.

Based on (11), $L$ scales with the number of activated antennas in (2), therefore, larger number of transmit antennas will result in higher system throughput. Moreover, (5), (6), and (7) show that the lower access distance (distance between UE and transmit antenna) provides lower path loss and interference-level. Therefore, by distributing all transmit antennas throughout the cell (RRUs), the probability of access distance will be increased, since UEs are randomly distributed, which consequently would result in higher system throughput.

The minimum of $P_{\mathrm{tx}}$ is achievable by reducing the transmission power. Therefore, according to (15) and its power sharing criteria (per central and distributed-antenna power constraints), by increasing the number of RRUs, the $P_{\mathrm{tx}}$ per antennas will be reduced. Therefore, fully distributed antennas will result in the minimum $P_{\mathrm{tx}}$. However, it should be noted that, since the total number of deployed antennas is variable, while the coverage area of the network is fixed, the maximum transmit power for all scenarios is constant (i.e., $46 \mathrm{dBm}$ ), in order to keep the coverage area acceptable/constant. Therefore, analytical analysis show that, by increasing the number of antennas and fully distributing them throughout the cell, the maximum of EE can be achieved.

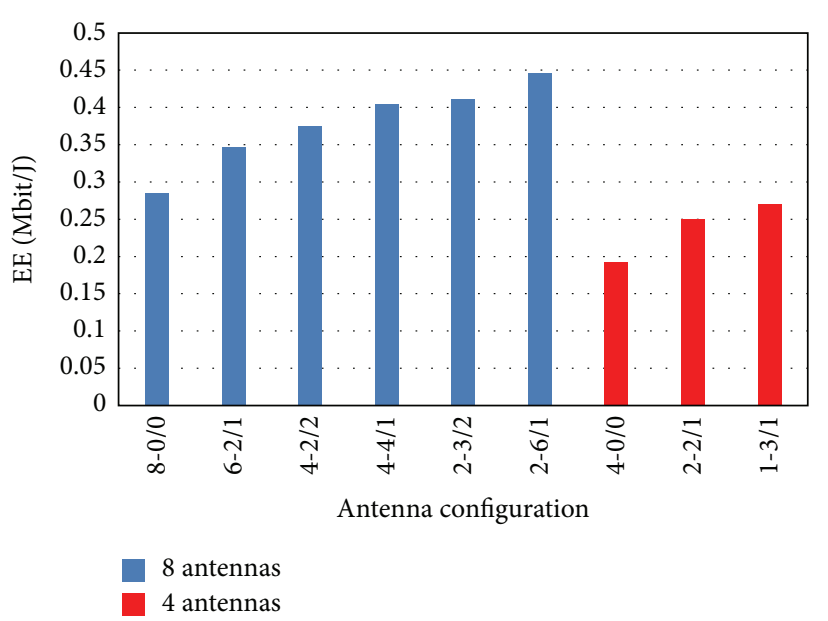

Figure 3: EE of CAS and DAS with different number of antennas/RRUs. High load scenario and $B_{w}=1.4 \mathrm{MHz}$.

Step 2 (optimal number of antennas for power-saving maximization (minimizing the total daily consumed power)). According to (8), the minimum power consumption, $P_{\text {tot }}^{\text {macro-BS }}$, can be achieved by reducing the number of activated antennas (minimizing the dynamic part of consumed power). For this purpose, we calculate the total consumed power according to (8) and set an EE degradation constraint based on the cell-load.

Note that the maximization of EE and power-saving depends on the number of activated antennas. The minimum of $P_{\text {tot }}^{\text {macro-BS }}$ can be achieved by reducing $N_{\text {ant }}^{\text {active }}$, and the maximum of EE can be achieved by increasing $N_{\text {ant }}^{\text {active }}$. By these means, $N_{\text {ant }}^{\text {active }}$ is the interconnected variable in the aforementioned two steps, and the optimal number of $N_{\text {ant }}^{\text {active }}$ is the solution to our problem. Therefore, number of activated antennas has to be reduced in order to jointly maximize the $\mathrm{EE}$ and power-saving in different traffic loads (by making tradeoff between EE degradation and power-saving). Finally, the amount of saved power by the proposed method can be calculated by solving (10).

\section{Result and Discussion}

4.1. Comparison of Energy Efficiency of DAS and CAS. Figure 3 compares the performance of different DAS configurations with CAS (8-0/0 and 4-0/0) in terms of EE. The results are shown for the high load scenario and to speed up the simulations, the smallest channel bandwidth of $B_{w}=$ $1.4 \mathrm{MHz}$ is considered. The simulation results show that in CAS by doubling the number of transmit antennas the EE increased by $48 \%$. While in DAS the improvement of EE highly depends to the number of RRUs, where the larger number of RRUs provides the higher EE. Because, in contrast to CAS, where all antennas are colocated at the central BS, by increasing the number of antennas and RRUs, more transmit antennas are distributed throughout the cell which results in more uniform cell coverage. Moreover, in this case, the access distance between UE and BS is reduced; therefore, lower 


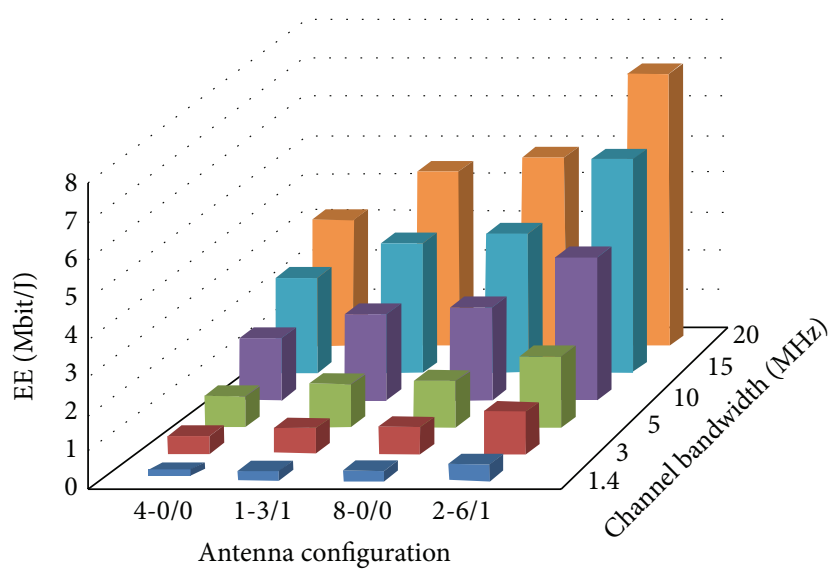

FIgure 4: Comparison of EE of DAS and CAS in terms of different antenna configurations and channel bandwidth. High load scenario.

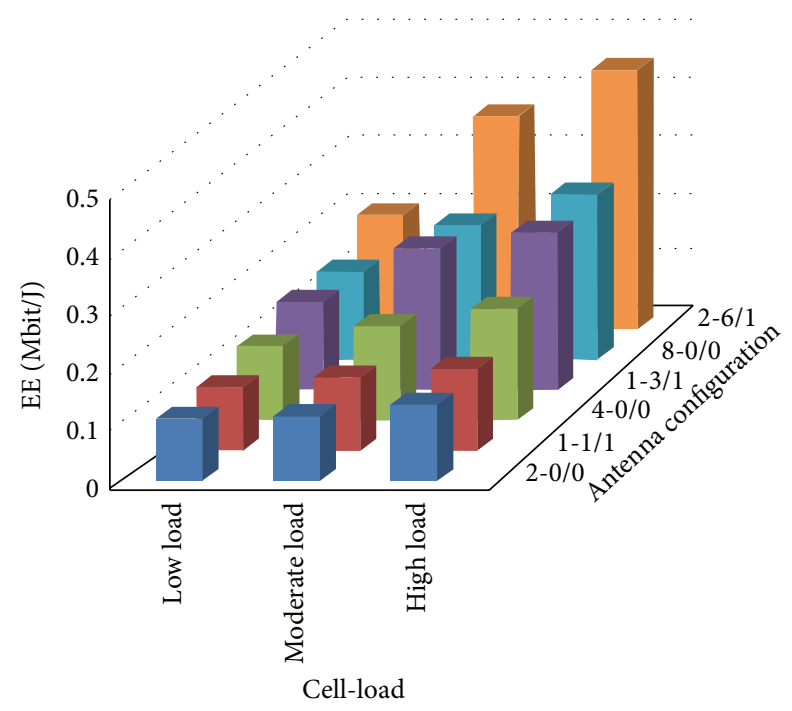

FIgURE 5: Comparison of cell throughput in three different cell-load scenarios and antenna configurations. $B_{w}=1.4 \mathrm{MHz}$.

power is required for downlink transmission where results in lower path loss and interference. Among the different antenna deployments, 2-6/1 and 1-3/1 (where all transmit antennas are fully distributed) provide best EE for DAS and considerably outperform CAS by $57 \%$ and $40 \%$, respectively.

Results of Figure 4 are provided in the high load scenario where EE of DAS and CAS is evaluated under different antenna configurations and channel bandwidths. For DAS the $2-6 / 1$ and $1-3 / 1$ were chosen as they provided the best performance. The results show that by increasing the channel bandwidth, the EE increases as well and leads to the higher EE gap between DAS and CAS. Interestingly, it can be seen that DAS with 4 transmit antennas (1-3/1) performs close to CAS with 8 transmit antennas (8-0/0), while the network considerably consumes less energy (this is because 4 PAs are switched-off).

Figure 5 compares EE in different cell-load scenarios where different antenna configurations are applied. The result
TABLE 2: EE degradation and power-saving in different antenna switching-off modes.

\begin{tabular}{lcc}
\hline \multirow{2}{*}{ Antenna muting mode } & \multicolumn{2}{c}{ EE degradation (\%) } \\
& Low load & Moderate load \\
\hline $2-6 / 1 \Rightarrow 1-3 / 1$ & 22.8 & 33.4 \\
$1-3 / 1 \Rightarrow 1-1 / 1$ & 28.4 & 48.2 \\
$2-6 / 1 \Rightarrow 1-1 / 1$ & 44.7 & 65.5 \\
\hline
\end{tabular}

demonstrates the dependency of DAS to the cell-load and number of RRUs. DAS plays significant role to improve $\mathrm{EE}$ in congested networks especially where the number of antennas is high. Because, more RRUs are distributed among the cell, which results in more uniform cell coverage, less path loss, and lower interference, while, in the presence of low number of users, there is no significant gap between EE of DAS and CAS. Moreover, it can be seen that in this case by increasing the number of antennas no significant $\mathrm{EE}$ gain can be achieved.

In this regard, 2-6/1 configuration is selected for high load scenario since it provides the best performance for DAS and significantly surpasses the CAS (8-0/0) in terms of EE by $56.6 \%$. Moreover, a comparison between two DAS configurations with 8 and 4 transmit antennas reveals that in high load scenario by switching off 4 transmit antennas (2-6/1 to $1-3 / 1$ ) the system throughput degrades by $60 \%$. Therefore, the 2-6/1 configuration is selected as the best DAS configuration for high load scenario.

Toward selecting the best DAS configurations (sleep modes) for low and moderate load scenarios, Table 2 presents the amount of EE degradation in different sleep modes. The results reveal that, in contrast to the congested networks, in the presence of lower number of users, the dependency of EE to the number of activated antennas is lower. It should be mentioned that the system throughput (data rate) scales with parameters such as number of transmit antennas, available bandwidth, number of activated users, and degree of SINR. Therefore, in the presence of a small number of users, few number of antennas could also provide the required service. On the other hand, it is clear that the power consumption degrades proportionally with reducing the number of antennas. So far, a cross comparison of results reveals that a good DAS configuration for moderate load scenario is $1-3 / 1$ where 4 antennas are switched off and EE degrades by $33.4 \%$. On the other hand, 1-1/1 configuration could provide an optimal performance for low load scenario where 6 antennas are switched off and only 28.4\% of EE is degraded (versus moderate load). However, in order to find the optimal number of antennas (DAS configuration) for two sleep modes, the following subsection considers the amount of power consumption for the aforementioned cases in detail and by making tradeoff between EE degradation and energy saving concludes the results.

4.2. Energy Saving. The goal of this paper is to obtain the optimal number of activated antennas in the sleep modes in order to maximize the EE and power-saving in DAS. In this 
TABLE 3: Power consumption of different DAS configurations.

\begin{tabular}{|c|c|c|c|c|c|c|c|}
\hline \multirow{3}{*}{ Parameter } & \multirow{3}{*}{ Unit } & \multicolumn{2}{|c|}{ High load } & \multicolumn{2}{|c|}{ Moderate load } & \multicolumn{2}{|c|}{ Low load } \\
\hline & & \multicolumn{2}{|c|}{$2-6 / 1$} & \multicolumn{2}{|c|}{$1-3 / 1$} & \multicolumn{2}{|c|}{$1-1 / 1$} \\
\hline & & CAS (2) & DAS (6) & CAS (1) & DAS (3) & CAS (1) & DAS (1) \\
\hline Max transmit rms power & [Watt] & 20 & 4 & 20 & 7 & 20 & 7 \\
\hline Max transmit rms power & {$[\mathrm{dBm}]$} & 43 & 36 & 43 & 38.5 & 43 & 38.5 \\
\hline Power-added efficiency & {$[\%]$} & 38.8 & 28.5 & 38.8 & 28.5 & 38.8 & 28.5 \\
\hline$P_{\mathrm{PA}}$ & [Watt] & 51.6 & 14.1 & 51.6 & 24.61 & 51.6 & 24.61 \\
\hline$P_{\mathrm{RF}}$ & [Watt] & 10.9 & 5.4 & 10.9 & 5.4 & 10.9 & 5.4 \\
\hline$P_{\mathrm{BB}}$ & [Watt] & 14.8 & 13.6 & 14.8 & 13.6 & 14.8 & 13.6 \\
\hline DC-loss & {$[\%]$} & 6 & 6.4 & 6 & 6.4 & 6 & 6.4 \\
\hline MS-loss & [\%] & 7 & 7.2 & 7 & 7.2 & 7 & 7.2 \\
\hline Cooling-loss & [\%] & 9 & 0 & 9 & 0 & 9 & 0 \\
\hline Total 1 antenna & [Watt] & 97.04 & 38.11 & 97.04 & 50.21 & 97.04 & 50.21 \\
\hline Number of antennas & $\#$ & 2 & 6 & 1 & 3 & 1 & 1 \\
\hline Total $N$ antenna & [Watt] & 194.1 & 228.64 & 97.04 & 150.62 & 97.04 & 50.21 \\
\hline Total power cons./sector & [Watt] & \multicolumn{2}{|c|}{422.72} & \multicolumn{2}{|c|}{247.66} & \multicolumn{2}{|c|}{147.25} \\
\hline Number of sectors & \# & \multicolumn{2}{|c|}{3} & \multicolumn{2}{|c|}{3} & \multicolumn{2}{|c|}{3} \\
\hline Total power cons./cell & [Watt] & \multicolumn{2}{|c|}{1268.17} & \multicolumn{2}{|c|}{743} & \multicolumn{2}{|c|}{441.75} \\
\hline
\end{tabular}

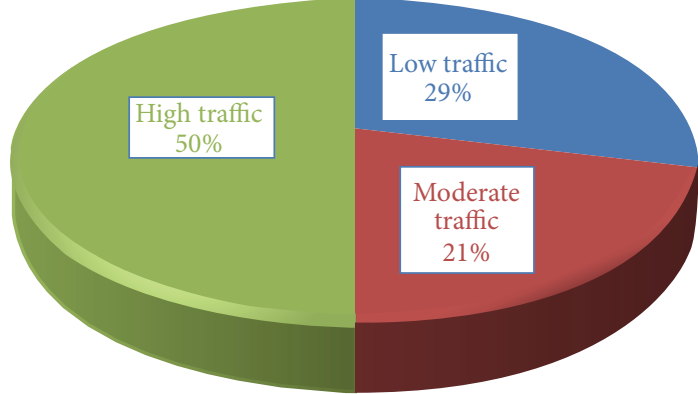

Figure 6: Percentage for each category of daily traffic.

regard, Figure 6 describes the percentage for each category of daily traffic. In addition, according to (8), Table 3 summarizes and compares the power consumption of different DAS configurations proposed in the present study. The last row of Table 3 gives the consumed power per hour for each selected DAS configuration in three different cell-load scenarios. The results show a power-saving of $41 \%$ and $66 \%$ for the two proposed sleep modes, moderate and low load scenarios, respectively (versus high load scenario).

Finally, we discuss aspects of the total daily energy saving that can be achieved by our proposed scheme. To do so, according to (10), the following briefly computes the daily power consumption for two cases.

(1) During normal operation (without switch-off scheme), where 2 antennas are located at the central BS and 6 antennas are distributed (RRUs) over 24 hours. Therefore,

$$
\begin{aligned}
P_{\text {Cons }}^{\text {day }} & =\left(N_{\text {ant }}^{\text {active }} \times P\right) \times \text { day-time } \\
& =126817 \times 24=30.44 \mathrm{~K} \text { Watt } / \text { day } .
\end{aligned}
$$

(2) During the proposed DAS switch-off scheme, during moderate load scenario, half of the total antennas are activated (one in central BS and 3 RRUs) for five hours, whereas during low load scenario, number of antennas reduce to one in the center and one RRU, as described in Table 3. Therefore,

$$
\begin{aligned}
P_{\text {Cons }}^{\text {day }} & =12(126817)+5(743)+7(441.75) \\
& =22.03 \mathrm{~K} \text { Watt } / \text { day } .
\end{aligned}
$$

The results show that, by applying the proposed scheme to the cellular system, the amount of energy consumption can be reduced by $8.41 \mathrm{~K}$ Watt per day, which means that $27.63 \%$ of the network power can be saved.

\section{Conclusion}

In this paper, the performance of different DAS configurations has investigated in terms of energy efficiency and compared with the CAS where all antennas are colocated at the central base station. It has shown that by distributing all transmitter antennas throughout the cell significant energy efficiency can be achieved versus CAS. Moreover, results have revealed high dependency of DAS to the cell load and number of RRUs, where DAS can provide significant EE gain in congested networks. Finally, based on the tradeoff between EE and power-saving, two DAS configurations (sleep modes) have been proposed for moderate and low load scenarios. The proposed methodology achieved $27.63 \%$ power-saving while guaranteeing a high capacity of data.

\section{Conflict of Interests}

The authors declare that there is no conflict of interests regarding the publication of this paper. 


\section{Acknowledgment}

The authors acknowledge the financial contribution from Universiti Kebangsaan Malaysia, Grant Ref. no. ETP-2013072 (Economic Transformation Programme) for the publication of this work.

\section{References}

[1] M. Etoh, T. Ohya, and Y. Nakayama, "Energy consumption issues on mobile network systems," in Proceedings of the International Symposium on Applications and the Internet (SAINT '08), pp. 365-368, August 2008.

[2] G. Fettweis and E. Zimmermann, "ICT energy consumptiontrends and challenges," in Proceedings of the 11th International Symposium on Wireless Personal Multimedia Communications, pp. 1-6, 2008.

[3] Z. Hasan, H. Boostanimehr, and V. K. Bhargava, "Green cellular networks: a survey, some research issues and challenges," IEEE Communications Surveys and Tutorials, vol. 13, no. 4, pp. 524540, 2011.

[4] X.-H. You, D.-M. Wang, B. Sheng, X.-Q. Gao, X.-S. Zhao, and M. Chen, "Cooperative distributed antenna systems for mobile communications," IEEE Wireless Communications, vol. 17, no. 3, pp. 35-43, 2010.

[5] O. Onireti, F. Héliot, and M. A. Imran, "On the energy efficiency-spectral efficiency trade-off of distributed MIMO systems," IEEE Transactions on Communications, vol. 61, no. 9, pp. 3741-3753, 2013.

[6] C. He, G. Y. Li, B. Sheng, and X. You, "Energy and spectral efficiency of distributed antenna systems," in Proceedings of the IEEE Wireless Communications and Networking Conference (WCNC '13), pp. 3225-3229, IEEE, April 2013.

[7] Q. Wang, D. Jiang, J. Jin, G. Liu, Z. Yan, and D. Yang, "Application of BBU+RRU based CoMP system to LTEadvanced," in Proceedings of the IEEE International Conference on Communications Workshops (ICC '09), pp. 1-5, June 2009.

[8] R. Heath, S. Peters, Y. Wang, and J. Zhang, "A current perspective on distributed antenna systems for the downlink of cellular systems," IEEE Communications Magazine, vol. 51, no. 4, pp. 161167, 2013.

[9] W. Choi and J. G. Andrews, "Downlink performance and capacity of distributed antenna systems in a multicell environment," IEEE Transactions on Wireless Communications, vol. 6, no. 1, pp. 69-73, 2007.

[10] R. W. Heath, T. Wu, Y. H. Kwon, and A. C. Soong, "Multiuser MIMO in distributed antenna systems with out-of-cell interference," IEEE Transactions on Signal Processing, vol. 59, no. 10, pp. 4885-4899, 2011.

[11] G.-H. Chen, C.-M. Yu, and C.-C. Huang, "A simulation study of a distributed antenna-based CDMA system," in Proceedings of the 7th IEEE International Symposium on Personal, Indoor and Mobile Radio Communications (PIMRC '96), vol. 2, pp. 517-521, Taipei, Taiwan, October 1996.

[12] L. Dai, "Distributed antenna system: performance analysis in multi-user scenario," in Proceedings of the 42nd Annual Conference on Information Sciences and Systems (CISS '08), pp. 85-89, Princeton, NJ, USA, March 2008.

[13] A. J. Paulraj, D. A. Gore, R. U. Nabar, and H. Bölcskei, "An overview of MIMO communications-a key to gigabit wireless," Proceedings of the IEEE, vol. 92, no. 2, pp. 198-217, 2004.
[14] R. Heath, T. Wu, Y. H. Kwon, and A. C. Soong, "Multiuser MIMO in distributed antenna systems," in Proceedings of the 44th Asilomar Conference on Signals, Systems and Computers (ASILOMAR '10), pp. 1202-1206, 2010.

[15] S. Schwarz, R. W. Heath, and M. Rupp, "Multiuser MIMO in distributed antenna systems with limited feedback," in Proceedings of the IEEE Globecom Workshops (GC Wkshps '12), pp. 546-551, IEEE, 2012.

[16] S. Schwarz, R. W. Heath Jr., and M. Rupp, "Single-user MIMO versus multi-user MIMO in distributed antenna systems with limited feedback," EURASIP Journal on Advances in Signal Processing, vol. 2013, article 54, 2013.

[17] S. Cui, A. J. Goldsmith, and A. Bahai, "Energy-efficiency of MIMO and cooperative MIMO techniques in sensor networks," IEEE Journal on Selected Areas in Communications, vol. 22, no. 6, pp. 1089-1098, 2004.

[18] X. Ge, C. Cao, M. Jo, M. Chen, J. Hu, and I. Humar, "Energy efficiency modelling and analyzing based on multi-cell and multi-antenna cellular networks," KSII Transactions on Internet and Information Systems, vol. 4, no. 4, pp. 560-574, 2010.

[19] T. Zhang, C. Zhang, L. Cuthbert, and Y. Chen, "Energy efficient antenna deployment design scheme in distributed antenna systems," in Proceedings of the IEEE 72nd Vehicular Technology Conference Fall (VTC '10), pp. 1-5, Ottawa, Canada, September 2010.

[20] H. Kim, S. R. Lee, C. Song, and I. Lee, "Optimal power allocation for energy efficiency maximization in distributed antenna systems," in Proceedings of the IEEE International Conference on Communications (ICC '13), pp. 5769-5773, June 2013.

[21] C. He, B. Sheng, P. Zhu, and X. You, "Energy efficiency and spectral efficiency tradeoff in downlink distributed antenna systems," IEEE Wireless Communications Letters, vol. 1, no. 3, pp. 153-156, 2012.

[22] C. He, B. Sheng, P. Zhu, X. You, and G. Y. Li, "Energyand spectral-efficiency tradeoff for distributed antenna systems with proportional fairness," IEEE Journal on Selected Areas in Communications, vol. 31, no. 5, pp. 894-902, 2013.

[23] M. Trivellato, F. Boccardi, and F. Tosato, "User selection schemes for MIMO broadcast channels with limited feedback," in Proceedings of the IEEE 65th Vehicular Technology Conference (VTC '07), pp. 2089-2093, April 2007.

[24] E. Björnson, M. Bengtsson, and B. Ottersten, "Receive combining vs. multistream multiplexing in multiuser MIMO systems," in Proceedings of the IEEE Swedish Communication Technologies Workshop (Swe-CTW '11), pp. 103-108, Stockholm, Sweden, October 2011.

[25] N. Jindal, "Antenna combining for the MIMO downlink channel," IEEE Transactions on Wireless Communications, vol. 7, no. 10, pp. 3834-3844, 2008.

[26] G. Auer, O. Blume, V. Giannini et al., "D2.3: energy efficiency analysis of the reference systems, areas of improvements and target breakdown," EARTH, 2010.

[27] C. Mehlführer, J. C. Ikuno, M. Šimko, S. Schwarz, M. Wrulich, and M. Rupp, "The Vienna LTE simulators-enabling reproducibility in wireless communications research," EURASIP Journal on Advances in Signal Processing, vol. 2011, pp. 1-14, article 29, 2011.

[28] T. Chen, Y. Yang, H. Zhang, H. Kim, and K. Horneman, "Network energy saving technologies for green wireless access networks," IEEE Wireless Communications, vol. 18, no. 5, pp. 3038, 2011. 
[29] Huawei, "Opportunities for energy savings in LTE networks," in Proceedings of the 2010 3GPP TSG RAN WG1 Meeting, \#59bis, 2010. 

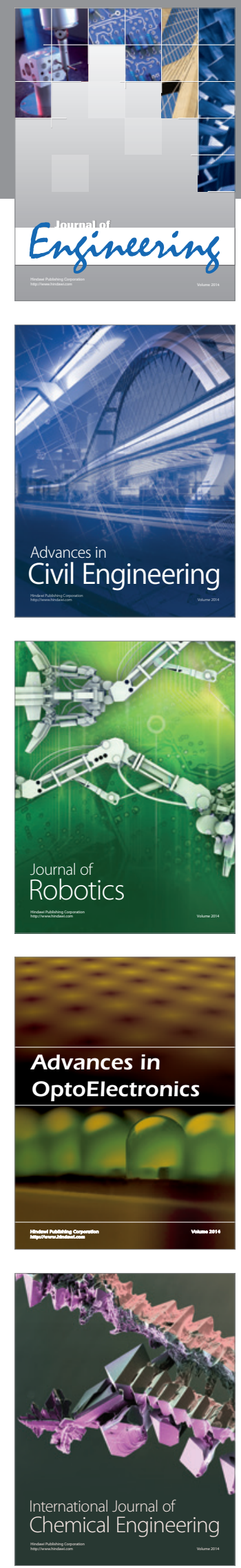

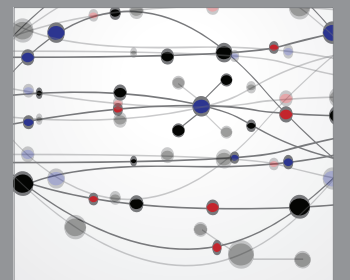

The Scientific World Journal
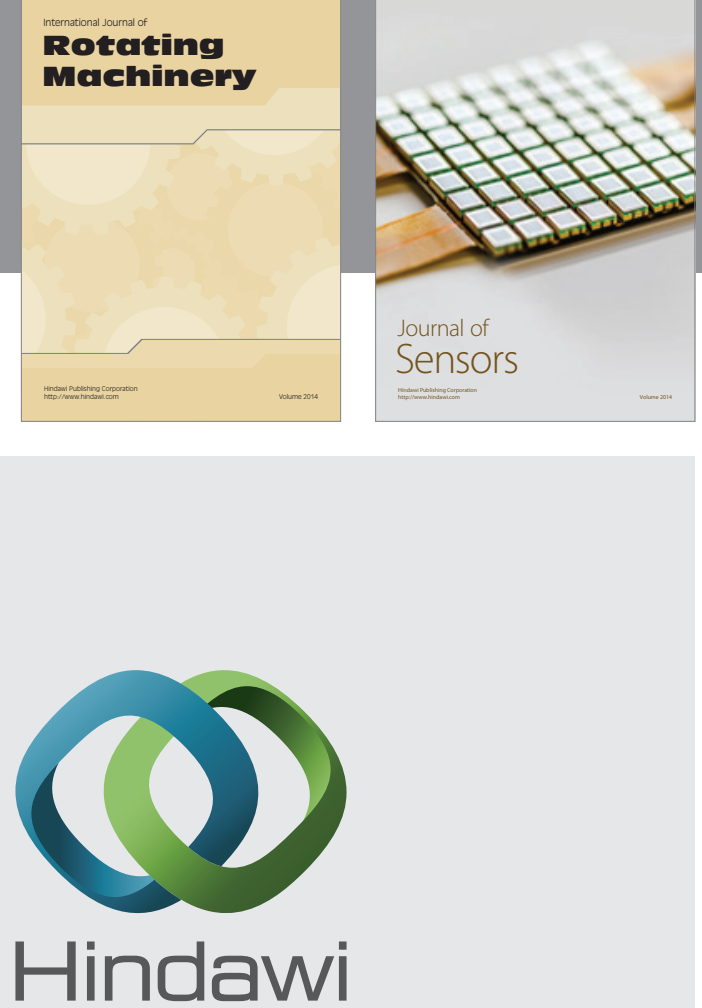

Submit your manuscripts at http://www.hindawi.com
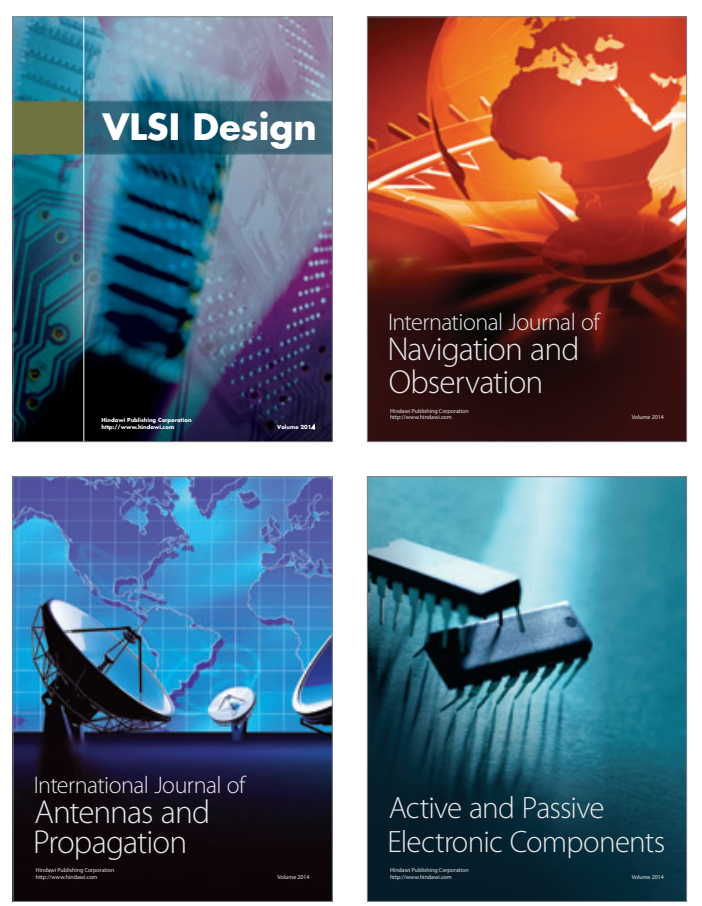
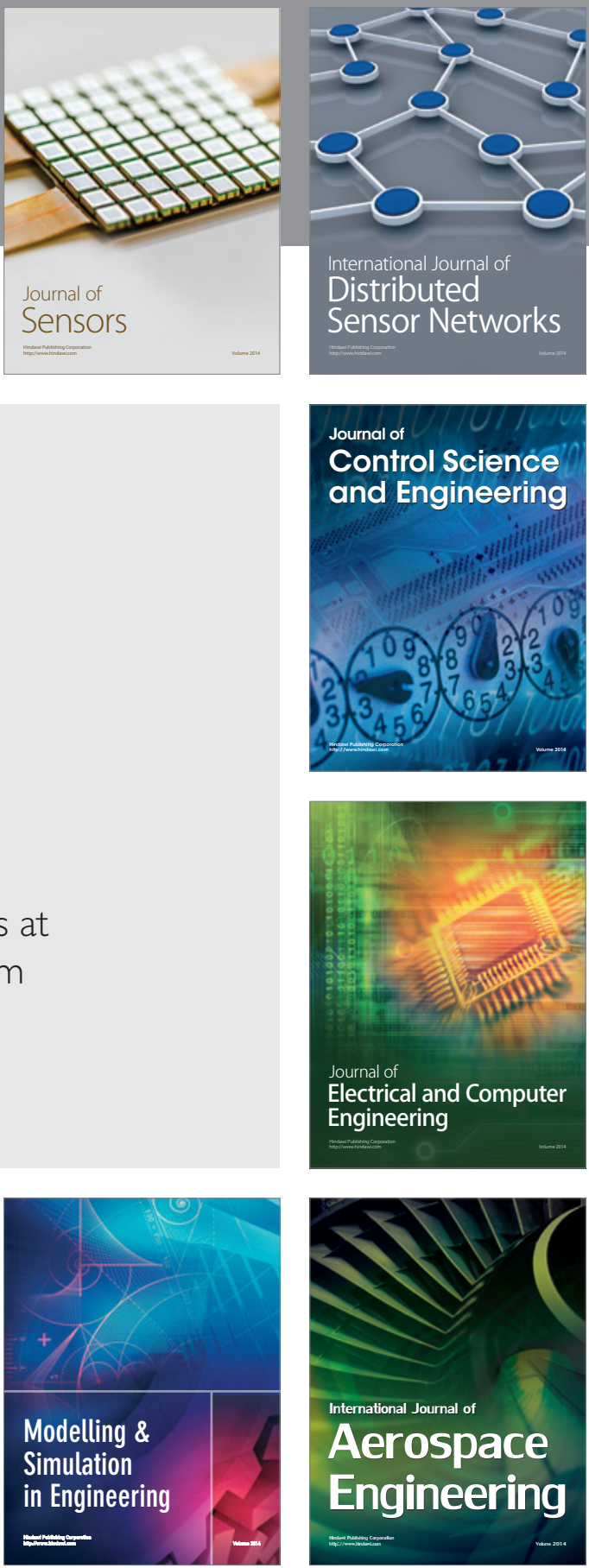

Journal of

Control Science

and Engineering
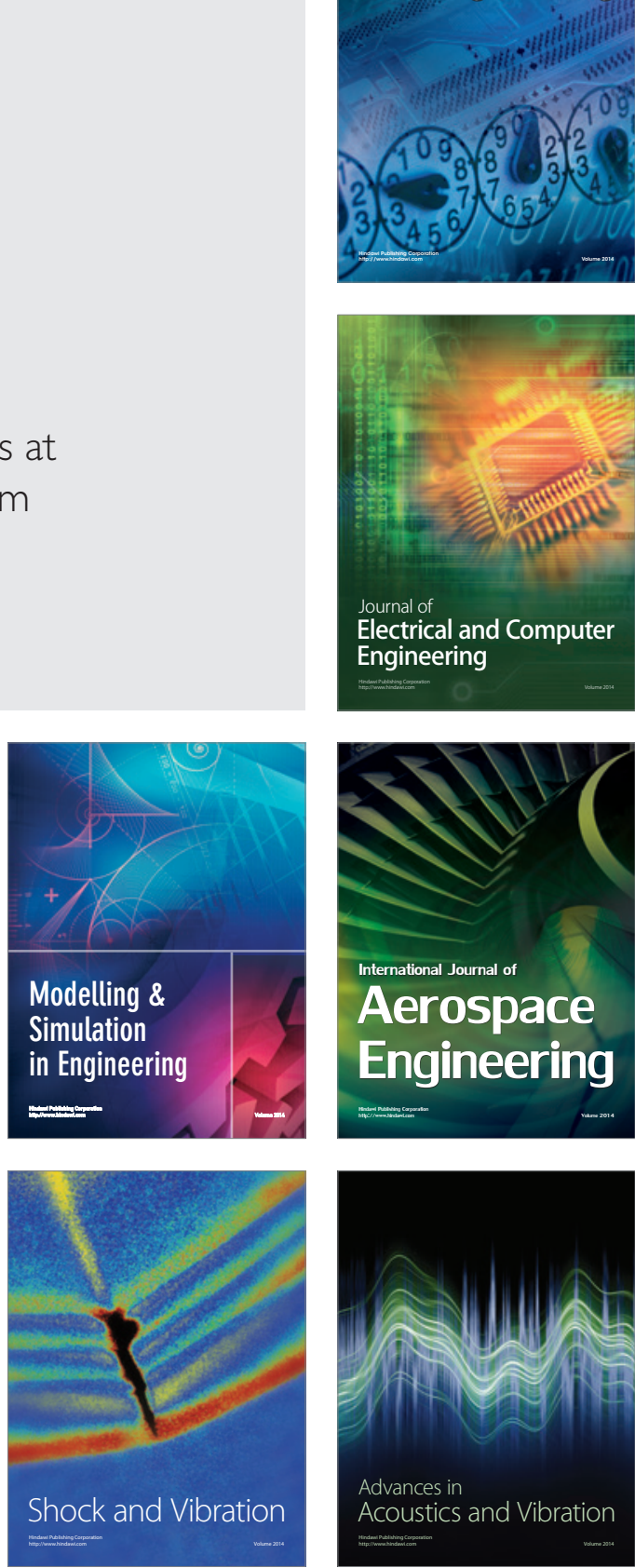